\title{
Ärztliche Bildung im Kontext von Veränderungen
}

\section{Iwan Rickenbacher}

Prof. Dr. phil., Erziehungswissenschaftler und

Kommunikationsberater

Schriftliche Fassung eines Vortrags im Rahmen der letztjährigen Plenarversammlung des SIWF.

Siehe dazu auch S. 296.
Korrespondenz:

Prof. Dr. phil. Iwan Rickenbacher Kommunikation und Beratung Gersauerstrasse 85 CH-6440 Brunnen

\section{Mein Standpunkt}

Ich bin nicht Mediziner. Als gelernter Erziehungswissenschaftler habe ich mich allerdings ein Berufsleben lang mit dem gesellschaftlichen Kontext von Bildung befasst, auch mit den Einwirkungen dieses Umfeldes auf Berufsbilder.

Im Lehrerinnen- und Lehrerberuf, im Umfeld, in dem ich mich lange Zeit bewegte, schwingen, so scheint es mir, heute noch frühe Erwartungen und Projektionen mit: Die Erwartungen an die Berufspioniere, die oft Mönche und Nonnen waren, die Ansprüche der Bürgerhäuser, die eigene Schulmeister beschäftigten, die Erwartungen der Gründer des Bundesstaates, die über beamtete Lehrpersonen feudale Gesellschaftsstrukturen zu überwinden trachteten.

Diese Formen und Ausprägungen des Berufes dringen bis in die Selbstwahrnehmung von Lehrpersonen, und dies auch heute, wo Berufsverbände für eine wissenschaftlich fundierte Berufsbildung aller Lehrpersonen, vom Kindergarten bis zum Gymnasium, eintreten. Logisch, dass die Ausbildung an Hochschulen angesiedelt wurde. Die aktuelle Auseinandersetzung um die Lehrerbildung an der Fachhochschule Nordwestschweiz zeigt aber in aller Deutlichkeit, dass die Verortung des Berufs zwischen Praxis und praxisleitender Theorie noch nicht abgeschlossen ist.

\section{Und die Ärztinnen und Ärzte?}

Kürzlich hörte ich einen Vortrag von Prof. Dr. med. Hubert Steinke, Direktor des Instituts für Medizingeschichte an der Universität Bern zu Ursprüngen und Entwicklungen im Arztberuf. Von den frühen Entwicklungen, zum Beispiel in der Chirurgie ausserhalb der Universitäten, von der anthropologischen Karriere der Medizinerausbildung an den Universitäten, von der Zusammenführung aller Arztberufe und deren zunehmenden Differenzierung in Spezialgebiete. Zurzeit sind es nichtärztliche Gesundheitsberufe, die ähnliche Entwicklungen erfahren, von der Pflege bis hin zur Psychotherapie und Physiotherapie, und dies mit Auswirkungen auf das Wirkungsfeld von Ärztinnen und Ärzten.

Die Treiber dieser Entwicklungen sind vielfältig. Sie reichen von Erwartungen der Patientinnen und Patienten über die Forderungen der Berufsvereinigungen, die Interessen der Industrien, der Politik bis hin zu den Profilierungen jener, die Fachkräfte im Gesundheitsbereich ausbilden.

Die Entwicklung medizinischer Berufe hin zu akademischen Abschlüssen widerspiegelt auch den
Stellenwert, den Gesundheit und Gesundheitserhaltung in unserer Gesellschaft einnehmen. Der Publizist Ludwig Hasler benennt Gesundheit als «Restgottheit des Jahrhunderts» und Medizin angesichts der fast uneinlösbaren Erwartungen als «Schicksalscasino».

Die Einbindung der Ausbildung im universitären Rahmen hat auch sekundäre Auswirkungen, die sich aus der Geschichte und aus der Positionierung der Hochschulen ergeben: der Ausrichtung auf Lehre und Forschung, der Ausrichtung der Hochschulen und ihrer Dozenten im internationalen Wettbewerb, der Rahmenbedingungen, unter denen die Hochschulen in den nächsten Jahren ihren Auftrag zu erfüllen haben.

\section{Veränderungen im Umfeld}

Im Frühjahr 2013 veröffentlichte der Bundesrat ein Dokument unter dem Titel «Gesundheit2020. Die gesundheitspolitischen Prioritäten des Bundesrates». Unter den vom Bundesrat identifizierten Schwerpunkten sind einige in besonderer Weise berufsrelevant:

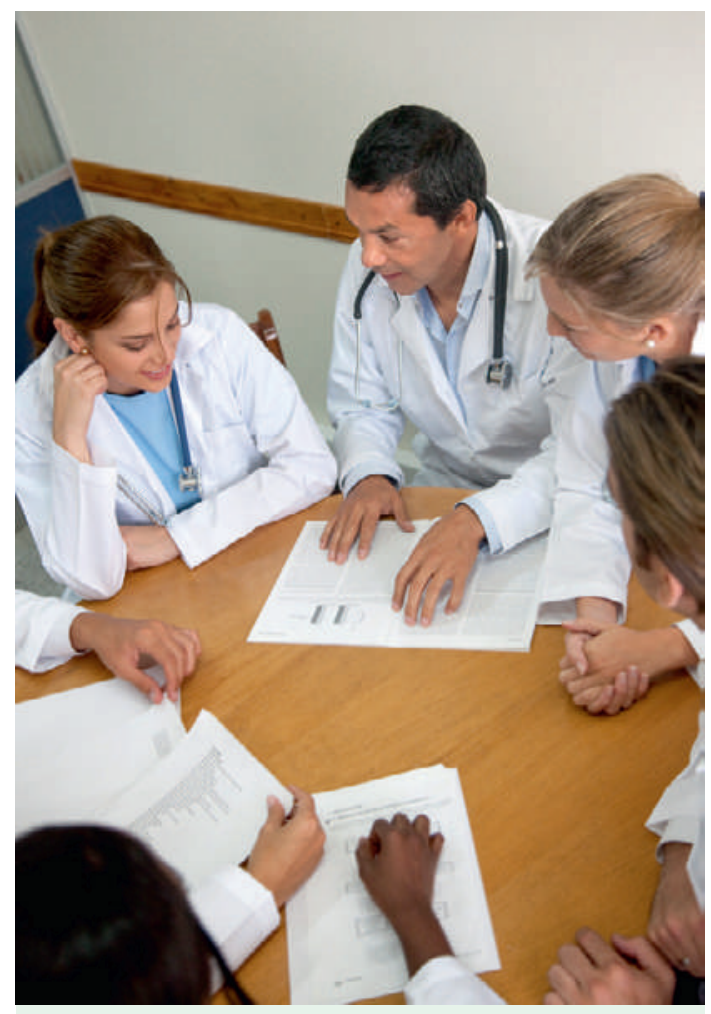

Die Zukunft der Medizin ist teamorientiert und interprofessionell. Doch Ärztinnen und Ärzte bleiben die Schlüsselpersonen im Gesundheitswesen. 
- Die Zunahme der chronischen Krankheiten als Folge der demographischen Veränderungen;

- Die Neuausrichtung der Versorgungsstrukturen von der Akutversorgung und stationären Behandlung hin zu Prävention, Langzeitversorgung und Palliativmedizin;

- Der Abbau von Doppelspurigkeiten, die Nutzung von Effizienzreserven auch unter Nutzung elektronischer Mittel, z.B. im Rahmen von E-Health;

- Die Schaffung von Transparenz über gute Datenbasen und die Erhöhung der Steuerbarkeit des Systems.

\section{«Grundbedingung eines Veränderungsprozesses ist,} die Notwendigkeit von Veränderungen bewusst

\section{zu machen.»}

Alle diese Ansatzpunkte sollen die Lebensqualität der Menschen sichern, die Chancengleichheit und die Selbstverantwortung stärken, die Versorgungsqualität erhöhen und die Koordination der Bemühungen verbessern.

Ein Blick in die Umsetzungspläne lässt erahnen, mit welchen Fragen sich jene Fachpersonen zu beschäftigen haben, die sich mit ärztlicher Aus- und Weiterbildung befassen:

- Die Einführung des elektronischen Patientendossiers;

- Neue Kompetenzen des Bundesrates im System TARMED;

- Die Stärkung der medizinischen Grundversorgung

- Neue Steuerungsmöglichkeiten im ambulanten Bereich;

- Die Zulassung nichtärztlicher Gesundheitsberufe als direkte Leistungserbringer.

Sie erfahren es bereits. Die Ausrichtung und Beurteilung ärztlicher Praxis auf Benchmarks, Best practice wird bedeutsamer. Die Einwirkungen regulatorisch tätiger Behörden werden spürbarer. Die ökonomischen Auswirkungen ärztlichen Tuns werden wichtiger. Die Abgrenzung zwischen ärztlicher und nichtärztlicher Intervention wird fliessender.

Im Übrigen stimmt die bundesrätliche Lagebeurteilung weitgehend mit der Roadmap der Akademien der Wissenschaften für ein nachhaltiges Gesundheitssystem Schweiz überein. Die Akademie betont in ihrem Massnahmenplan zudem die Sicherung einer genügenden Zahl gutausgebildeter Fachpersonen, «die am richtigen Ort zum Einsatz kommen», und unterstreicht, dass

- die Curricula und Abschlusskompetenzen des Fachpersonals an die Bedürfnisse und Prioritäten der Bevölkerung anzupassen seien;
- die Aus- und Weiterbildungsprogramme sowie die entsprechenden Ausbildungsplätze auf die neuen Profile in der ambulanten und stationären Versorgung auszurichten seien;

- die Interaktion in der Aus-, Weiter- und Fortbildung der Gesundheitsfachleute, zwischen Ärzten, Pflegefachpersonen und Therapeuten $\mathrm{zu}$ verstärken sei;

- attraktivere Arbeitsbedingungen für das Gesundheitspersonal bereitzustellen seien;

- Angebote für Wiedereinsteigerinnen und -einsteiger vorzusehen seien;

- Modelle der interdisziplinären Teamarbeit zu entwickeln und erproben seien.

Zu bedenken ist zudem, dass die neue Spitalfinanzierung bezüglich der ärztlichen und nichtärztlichen Aus- und Weiterbildung an den Spitälern eine neue Situation schafft. Die entsprechenden Kosten entfallen auf unterschiedliche Kostenträger: Die nichtärztliche Aus- und Weiterbildung auf die obligatorischen Krankenversicherungen, die ärztliche auf die Kantone.

\section{Wer etwas verändern will, verändern muss}

Viele der Veränderungen, die eben genannt worden sind, werden bereits eingeleitet oder treten nächstens ein. Die Wirkung muss vorab mit Menschen erzielt werden, die zu anderen Rahmenbedingungen und Konditionen ausgebildet worden sind. Dies geht nicht, ohne vorherrschende Mentalitäten zu verändern.

Grundbedingung eines Veränderungsprozesses ist, die Notwendigkeit von Veränderungen bewusst $\mathrm{zu}$ machen, mindestens bei jenen Zielgruppen, die sich nicht in die Pensionierung zu retten glauben. Ich habe den Eindruck, dass es am Problembewusstsein unter den Ärztinnen und Ärzten nicht mehr mangelt.

Mit dem SIWF verfügt die Ärzteschaft über eine hervorragende Innovationsagentur, welche die wichtigsten Akteure in der Aus- und Weiterbildung der Ärztinnen und Ärzte vereinigt. Dieses Netzwerk ist unabdingbar, um Veränderungsbedarf zu orten und Veränderungen möglichst flächendeckend und zeitgerecht umzusetzen.

Notwendig ist eine Vision, wie zum Beispiel die von Werner Bauer mitgestaltete Arbeit «die zukünftigen Berufsbilder von ÄrztInnen und Pflegenden» mit der These: «Die Zukunft der Medizin ist teamorientiert, interprofessionell und modular». An dieser Vision ist permanent weiter $\mathrm{zu}$ arbeiten, auch und nicht zuletzt im Rahmen des SIWF.

Es ist effizient, die Veränderungen über die Dozierenden und über die Ausbildner zu fördern. Zur didaktischen Fortbildung der Dozierenden gehört auch die Vermittlung der Veränderungsszenarien und der Vision.

Es ist die Vernetzung mit den Organisationen der Pflegenden zu suchen, mit den Organisationen an- 
derer Gesundheitsberufe, die in dieser teamorientierten, interprofessionellen und modularen Gesundheitswelt eine wichtige Rolle spielen, auch wenn die Interessen in Teilen divergieren.

Und dann ist nicht auszublenden, dass Veränderungen auch Gefühle auslösen, Ängste schüren, Widerstände wecken. Wenn es gelingt, den Ärztinnen und Ärzten bewusst zu machen, dass sie die Schlüsselpersonen im Gesundheitswesen bleiben, zumindest in den Augen der Patientinnen und Patienten und damit auch der Stimmberechtigten, dann sind sie eher bereit, Gewohnheiten aufzugeben und neue Wege zu gehen. Vielleicht braucht es noch die eine und andere Erfahrung anderer Stakeholder in Abstimmungen zum Beispiel, um bewusst zu machen, dass im Netzwerk jener, die ein effizientes Gesundheitssystem erhalten und entwickeln wollen, die Ärztinnen und Ärzte an vorderster Front mitzuwirken haben.
Sie, Mitglieder des SIWF, dem «Kompetenzzentrum der Schweiz rund um die ärztliche Weiter- und Fortbildung», so Ihre Deklaration, sind nicht die einzige Instanz, die Ärztinnen und Ärzte befähigen oder behindern, ihr Tun auf validierte Erkenntnisse auszurichten. Im Wissen darum gehört es wahrscheinlich zu Ihrer Aufgabe, auch die politisch-gesellschaftliche Kompetenz der Mediziner zu erhöhen, indem Sie selber den gesellschaftlichen Kontext analysieren, in dem Ärztinnen und Ärzte agieren und in dem Sie über Ihre angeschlossenen Verbände dort auf Entwicklungen Einfluss nehmen, wo ärztliches Tun wesentlich bestimmt wird.

Lange bevor im Schulbereich über das Konzept Geleitete Schule ein Teil der Entscheidungskompetenz von Behörden an die Lehrerschaft überging, habe ich im Lehrerseminar des Kantons Schwyz ziemlich radikale Formen der studentischen Mitbestimmung eingeführt, und dies mit der Absicht, we-

\section{«Es ist die Vernetzung mit den Organisationen der Pflegenden zu suchen, mit den Organisationen anderer Gesundheitsberufe.»}

Damit sind wir aber wieder bei Mentalitäten, die allenfalls zu verändern sind. Politische Forderungen $\mathrm{zu}$ formulieren und dann auch einzufordern, ist für viele Ärztinnen und Ärzte eher gewöhnungsbedürftig. Sie möchten sich als selbständige, dem eigenen Gewissen und der erlernten Kunst verpflichtete, auf das Wohl ihrer Patientinnen und Patienten ausgerichtete und dank ihrer Ausbildung unbestrittene Gesundheitsfachleute verstehen, deren Argumente für sich sprechen. Das waren Ärztinnen und Ärzte so wahrscheinlich nie. Sicher aber stets Akteure innerhalb von Politik und Gesellschaft und darum wohl beraten, auf jene Konditionen Einfluss zu nehmen, die in der Politik gestaltet werden und die für ihr ärztliches Handeln relevant sind. nigstens einen Teil der künftigen Lehrerschaft in die Lage zu versetzen, das Instrumentar demokratischer Einflussnahme aktiv zu nutzen. Denn ich fand, die Schule sei zu bedeutsam, um ihre Gestaltung Verwaltungsjuristen, Hirnforschern oder Soziologen zu überlassen.

Sie stehen angesichts der Gesundheitsökonomen, der Gesundheitsverwalter, der Naturwissenschaftler und Ingenieure vor ähnlichen Herausforderungen. Nachdem die Quacksalber weitgehend entlarvt sind, sollten wir kranke Menschen nicht dem Gutdünken von Technokraten überlassen. Die Menschen spüren das. Sie möchten ihre Ärztinnen und Ärzte. 